EPJ manuscript No.

(will be inserted by the editor)

\title{
Strange and Heavy Flavoured Hypernuclei in Chiral Soliton Models
}

\author{
Vladimir B.Kopeliovich and Andrei M.Shunderuk \\ Institute for Nuclear Research of Russian Academy of Sciences, Moscow 117312 \\ the date of receipt and acceptance should be inserted later
}

\begin{abstract}
The extention of the chiral soliton approach to hypernuclei - strange or heavy flavoured - becomes more reliable due to success in describing of other properties of nuclei, e.g. the symmetry energy of nuclei with atomic numbers up to $\sim 30$. The binding energies of the ground states of light hypernuclei with $S=-1$ have been described in qualitative agreement with data. The existence of charmed or beautiful hypernuclei and Theta-hypernuclei (strange, charmed or beautiful) with large binding energy is expected within same approach. -
\end{abstract}

PACS. 12.39.Dc, 21.60.Ev, 21.80.+a, 14.20.-c

\section{Main features of the chiral soliton approach.}

The chiral soliton approach (CSA) is based on few principles and ingredients incorporated in the truncated effective chiral lagrangian:

$$
\begin{aligned}
L^{e f f} & =-\frac{F_{\pi}^{2}}{16} \operatorname{Tr}\left(l_{\mu} l_{\mu}\right)+\frac{1}{32 e^{2}} \operatorname{Tr}\left[l_{\mu} l_{\nu}\right]^{2}+ \\
& +\frac{F_{\pi}^{2} m_{\pi}^{2}}{16} \operatorname{Tr}\left(U+U^{\dagger}-2\right)+\ldots,
\end{aligned}
$$

$l_{\mu}=\partial_{\mu} U U^{\dagger}, U \in S U(2)$ or $U \in S U(3)$ - unitary matrix depending on chiral fields, $m_{\pi}$ is the pion mass, $F_{\pi}$-pion decay constant, $e$ - the only parameter of the model.

The soliton (skyrmion) is coherent configuration of classical chiral fields, possessing topological charge (or winding number) identified with the baryon number $B$ (Skyrme, 1961). Important simplifying feature of this approach is that configurations with different baryon, or atomic numbers are considered on equal footing, when zero modes only are taken into account in the quantization procedure. Another feature is that baryons individuality is absent within the multiskyrmion, and can be recovered - as it is believed - due to careful consideration of the nonzero modes.

The observed spectrum of baryon states is obtained by means of quantization procedure and depends on their quantum numbers (isospin, strangeness, etc) and static characteristics of classical configurations. For the $B=1$ case this was made first in the paper 11. Masses, binding energies of classical configurations with baryon number $B \geq 2$, their moments of inertia $\Theta_{I}, \Theta_{J}, \Sigma$-term $(\Gamma)$, and some other characteristics of the chiral solitons contain implicitly information about interaction between baryons.
They are obtained usually numerically and depend on parameters of the model $F_{\pi}, e$ and masses of mesons which enter the mass term in the effective lagrangian 1 .

\section{Ordinary $(S=0)$ nuclei; symmetry energy as quantum correction}

In the $S U(2)$ case, which is relevant for description of nonstrange baryons and nuclei, the rigid rotator quantization model is most adequate when quantum corrections are not too large. The symmetry energy $E_{\text {sym }}=$ $b_{\text {sym }}(N-Z)^{2} /(2 A), b_{\text {sym }} \simeq 50 \mathrm{MeV}$, within chiral soliton approach is described mainly by the isospin dependent quantum correction

$$
\delta E_{I}=\frac{I(I+1)}{2 \Theta_{I}},
$$

$\Theta_{I} \sim A$ being isotopical moment of inertia, $I=(N-Z) / 2$ for the ground states of nuclei. The $S U(2)$ quantization method - simplest and most reliable - is used here according to [1]. The moment of inertia $\Theta_{I}$ grows not only with increasing number of colours, but also with increasing baryon number ( $\sim B$ approximately), therefore this correction decreases like $\sim 1 / B$ and such estimates become more selfconsistent for larger $B$.

In Fig.1 the differences of binding energies between states with integer isospins are shown, for the even-even or for the odd-odd nuclei (e.g. such differences for the $I=0$

1 It is of interest that baryon interaction potentials depend on the weak decay constant $F_{\pi}$ and Skyrme parameter $e$. This connection of weak and strong interaction properties apparently needs deeper understanding. 


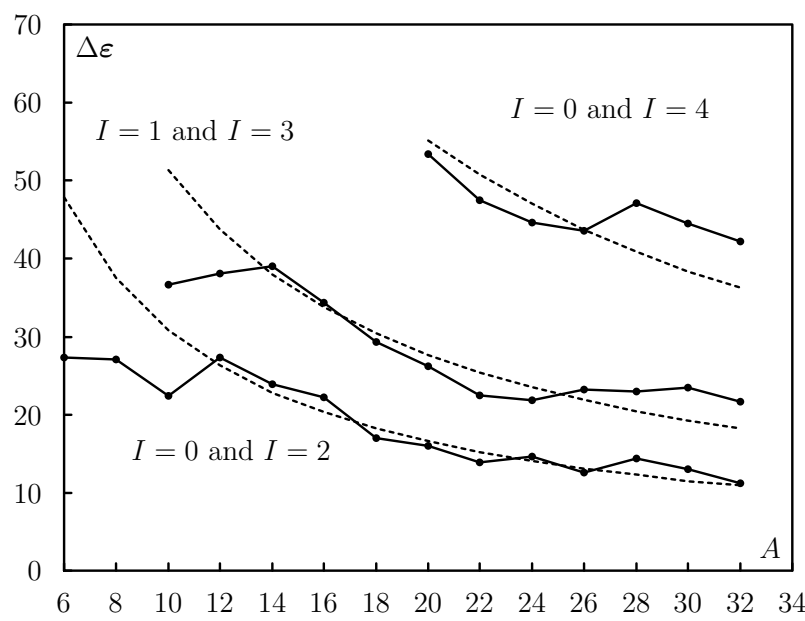

Fig. 1. The binding energies differences (in $\mathrm{MeV}$ ) for isotopes with even atomic numbers (integer isospins) for the "nuclear variant" of the model with rescaled constant, $e=3.0, F_{\pi}=$ $186 \mathrm{MeV}$ (black points connected with solid lines - experimental data, dashed lines - model calculations). The values of isospin of nuclei which binding energies differences are calculated, are indicated within the figure.

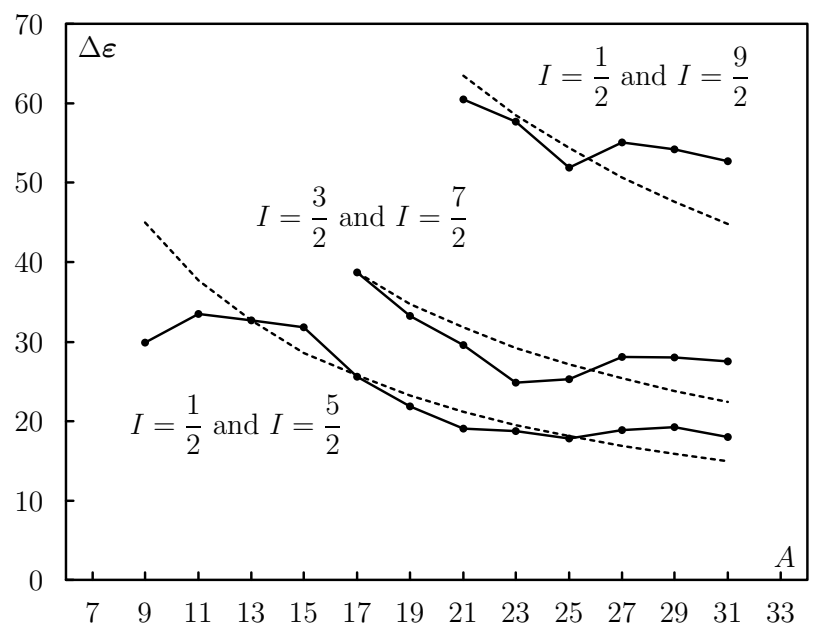

Fig. 2. The binding energies differences (in $M e V$ ) for isotopes with odd atomic numbers (half-integer isospins) for the rescaled, or "nuclear" variant of the model, $e=3.0, F_{\pi}=$ $186 \mathrm{MeV}$. Notations for experimental data and calculation results as in Fig.1. Both Fig.1 and Fig.2 are taken from the paper [2].

and $I=2$ states or for the $I=1$ and $I=3$ ), calculated with the help of formula (2) and for the value of the model parameter $e=3.0$ (for the $B=1$ case the value of $e$ is taken usually close to $e \simeq 4.1$ which allowed to describe the mass splittings of baryons octet and decuplet). Many uncertainties and some specific corrections introduced in the nuclear mass formula are cancelled in such differences (see 2 for details and references). Similar differences for the odd-even or even-odd nuclei with half-integer isospins are shown in Fig.2. The differences of binding energies of other nuclei, besides those shown in Fig.1 and Fig.2, are also described well, see 2. The change of the model parameter $e$ is a natural way to take into account effectively the nonzero modes - breathing, vibration - which lead to the increase of dimensions of multiskyrmions (the natural unity of length in the model is $\sim 1 /\left(F_{\pi} e\right)$ ). Recently similar procedure has been used for description of the ${ }^{6} \mathrm{Li}$ nucleus [3] properties. The change of the pion decay constant $F_{\pi}$, also made in [3], is much more limited since it is directly measurable quantity, via pion decay.

The mass and baryon number distributions of multiskyrmions have shell-like form [4, at variance from the real ones. However, skyrmions are easily deformable objects, as previous experience has shown [5] and recently has been observed, e.g. for the $B=7$ multiskyrmion 6 . Therefore, one can hope that transition to realistic shape of the mass distribution could proceed without large increase of the energy. Moreover, the important result obtained recently numerically by Battye, Manton and Sutcliffe [7] is that at large baryon numbers and large enough value of the chiral symmetry breaking mass in the lagrangian (1) the transition to more realistic alpha-cluster shape takes place.

The quantum correction due to collective rotation of the multiskyrmion in usual space equals to $\delta E_{J}=J(J+$ $1) /\left(2 \Theta_{J}\right)$, where $J$ is spin of the nucleus. It is technically complicated problem to define allowed values of $J$ for the classical configuration with definite symmetry properties. The experimentally observed value of spin of the nucleus' ground state not always can be obtained when quantization of the lowest in energy classical configuration is made [8 9. However, due to rich landscape of the classical energy local minima with the energy not much different from the lowest one, but different symmetry properties of the chiral field configurations 4, quantization of one of them could give the desired value of spin (the $B=7$ case has been considered recently in details in 6]), and we make here in fact a natural assumption that it is always possible.

Since the orbital inertia $\Theta_{J}$ is considerably greater than isotopical one $\Theta_{I}\left(\Theta_{J} \geq B \Theta_{I}\right)$, this correction is not important for large enough baryon numbers and is not included in 2] and here. The success of the CSA in description the differences of binding energies of known nuclei allows to make predictions for the binding energies of still unknown neutron-rich nuclides (some examples were considered in [2]) and to go further to the consideration of different kinds of hypernuclei.

\section{Strange hypernuclei $(S=-1)$; binding energies of ground states}

In the $S U(3)$ case invoking strangeness (or charm, beauty) the flavour symmetry breaking terms in the lagrangian

$$
\begin{aligned}
& L_{F S B}= \\
= & -\frac{F_{K}^{2} m_{K}^{2}-F_{\pi}^{2} m_{\pi}^{2}}{24} \operatorname{Tr}\left[\left(1-\sqrt{3} \lambda_{8}\right)\left(U+U^{\dagger}-2\right)\right]+\ldots
\end{aligned}
$$

play the crucial role in calculating the spectrum of states with different flavours (strangeness first of all). Some terms 
Table 1. The collective motion contributions to the binding energies of the isoscalar hypernuclei with unit flavour, strangeness or beauty, $S=-1$ or $b=-1$, in $M e v . \Delta \epsilon_{s, b}$, in $M e v$, are the changes of binding energies of lowest baryonic state with unit flavour, in comparison with usual nuclei with the same B-number. $\epsilon^{\text {tot }}$ is the total binding energy of the hypernucleus. Experimental values $\epsilon_{\text {exp }}^{t o t}$ are taken from 13] and [14. For beauty the first 3 columns correspond to $r_{b}=F_{B} / F_{\pi}=1.5$, and the last 3 - to $r_{b}=2$.

\begin{tabular}{lllllllllll}
\hline${ }_{\Lambda} A$ & $\omega_{s}$ & $\Delta \epsilon_{s}$ & $\epsilon_{s}^{t o t}$ & $\epsilon_{e x p, s}^{t o t}$ & $\omega_{b}^{r_{b}=1.5}$ & $\Delta \epsilon_{b}$ & $\epsilon_{b}^{t o t}$ & $\omega_{b}^{r_{b}=2}$ & $\Delta \epsilon_{b}$ & $\epsilon_{b}^{\text {tot }}$ \\
\hline 1 & 306 & - & - & - & 4501 & - & - & 4805 & - & - \\
${ }_{\Lambda}^{3} H$ & 289 & -3 & 5 & 2.35 & 4424 & 75 & 83 & 4751 & 53 & 61 \\
${ }_{\Lambda}^{5} H e$ & 287 & -6 & 33 & 31.4 & 4422 & 76 & 103 & 4749 & 54 & 81 \\
${ }_{\Lambda}^{7} L i$ & 282 & -3 & 29 & 37.2 & 4429 & 81 & 119 & 4744 & 59 & 97 \\
${ }_{\Lambda}^{9} B e$ & 291 & -13 & 40 & 62.5 & 4459 & 40 & 97 & 4773 & 31 & 88 \\
${ }_{\Lambda}^{11} B$ & 294 & -16 & 59 & - & 4478 & 21 & 96 & 4786 & 18 & 93 \\
${ }_{\Lambda}^{13} \mathrm{C}$ & 295 & -18 & 78 & 104 & 4488 & 10 & 106 & 4793 & 11 & 107 \\
\hline
\end{tabular}

Table 2. The binding energies of the isodoublets of hypernuclei with unit flavour, strangeness or beauty. Other notations and peculiarities as in Table 1. Experimental values are from 13 .

\begin{tabular}{lllllllll}
\hline$\Lambda A$ & $\omega_{s}$ & $\Delta \epsilon_{s}$ & $\epsilon_{s}^{t o t}$ & $\epsilon_{e x p}^{t o t}$ & & $\omega_{b}^{r_{b}=2}$ & $\Delta \epsilon_{b}$ & $\epsilon_{b}^{\text {tot }}$ \\
\hline${ }_{\Lambda}^{4} \mathrm{H}-{ }_{\Lambda}^{4} \mathrm{He}$ & 283 & -23 & 5.3 & $10.5 ;$ & 10.1 & 4735 & 52 & 80 \\
${ }_{\Lambda}^{6} \mathrm{He}-{ }_{\Lambda}^{6} \mathrm{Li}$ & 287 & -22 & 10.3 & $31.7 ;$ & 30.8 & 4752 & 40 & 72 \\
${ }_{\Lambda}^{8} \mathrm{Li}-{ }_{\Lambda}^{8} \mathrm{Be}$ & 288 & -20 & 36.5 & $46.0 ;$ & 44.4 & 4765 & 33 & 89 \\
${ }_{\Lambda}^{10} \mathrm{Be}-{ }_{1}^{10} \mathrm{~B}$ & 292 & -23 & 42 & $67.3 ;$ & 65.4 & 4778 & 20 & 85 \\
${ }_{\Lambda}^{12} \mathrm{~B}-{ }_{\Lambda}^{12} \mathrm{C}$ & 294 & -24 & 67 & $87.6 ;$ & 84.2 & 4788 & 11 & 103 \\
\hline
\end{tabular}

a) $\operatorname{Odd} B, J=1 / 2$

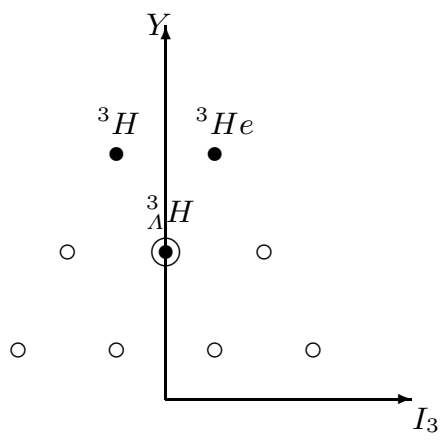

b) Even $B, J=0$

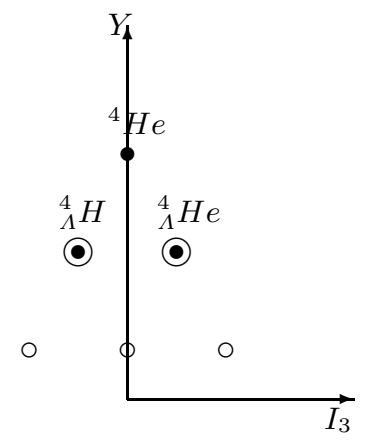

Fig. 3. (a) The location of the isoscalar state (shown by double circle) with odd $B$-number and $|F|=1$ in the upper part of the $\left(I_{3}-Y\right)$ diagram. (b) The same for isodoublet states with even $B$. The case of light hypernuclei ${ }_{\Lambda} H$ and ${ }_{\Lambda} H e$ is presented as an example. The lower parts of diagrams with $Y \leq B-3$ are not shown here.

proportional to the difference $F_{K}^{2}-F_{\pi}^{2}$ are omitted here (see 10,11,12 for details and references). Different quantization schemes have been used in literature: rigid rotator, soft rotator or bound state model.

The version of the bound state soliton model proposed by Callan, Klebanov, Westerberg (1985 - 1996) and modified for the flavour symmetry breaking case $\left(F_{K}>F_{\pi}\right)$ allows to calculate the binding energy differences of ground states between flavoured and unflavoured nuclei. Combined with few phenomenological arguments it is very successful in some cases of light hypernuclei.

Within bound state model (BSM) [10] (see also [11, 12 for details and Fig.3 where location of strange baryon

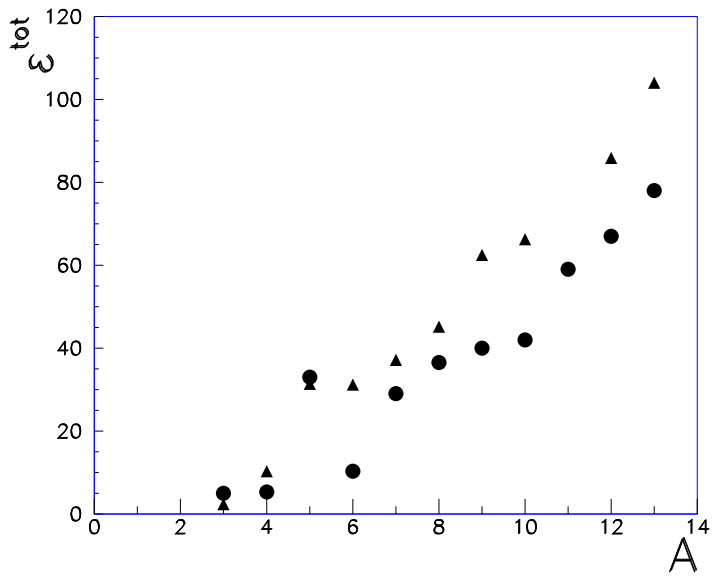

Fig. 4. The total binding energies (in $\mathrm{MeV}$ ) of the ground states of light $S=-1$ hypernuclei. Triangles - experimental data, full circles - the collective motion contribution.

states within minimal $S U(3)$ multiplets is shown)

$$
M=M_{c l}+\omega_{F}+\omega_{\bar{F}}+|F| \omega_{F}+\Delta M_{H F S}
$$

where flavour and antiflavour excitation energies

$$
\omega_{F}=N_{c} B(\mu-1) / 8 \Theta_{F}, \quad \omega_{\bar{F}}=N_{c} B(\mu+1) / 8 \Theta_{F},
$$

$\mu \simeq \sqrt{1+\bar{m}_{F}^{2} / M_{0}^{2}}, \bar{m}_{K}^{2}=m_{K}^{2} F_{K}^{2} / F_{\pi}^{2}-m_{\pi}^{2}$, and similar for $D$ or $B$ mesons, $M_{0}^{2} \simeq N_{c}^{2} B^{2} /\left(16 \Gamma \Theta_{F}\right), \Theta_{F}\left(\right.$ or $\left.\Theta_{K}\right)$, is the so called flavour moment of inertia for rotation of the skyrmion to "flavoured direction" - strange, or charmed, etc., the number of colours $N_{c}=3$ in all realistic calculations. The hyperfine splitting correction $\triangle M_{H F S}$ for the 
cases we consider here can be written in the form

$$
\begin{aligned}
\Delta M_{H F S} & =\frac{J(J+1)}{2 \Theta_{I}}+\left(\bar{c}_{F}-c_{F}\right) \frac{I_{F}\left(I_{F}+1\right)}{2 \Theta_{I}} \\
& +\left(c_{F}-1\right) \frac{\left[I_{r}\left(I_{r}+1\right)-I(I+1)\right]}{2 \Theta_{I}}
\end{aligned}
$$

with $I_{F}=|F| / 2=1 / 2$, and the hyperfine splitting constants $c_{F}$ and $\bar{c}_{F}$ given by

$$
c_{F}=1-\frac{\Theta_{I}}{2 \mu \Theta_{F}}(\mu-1) ; \quad \bar{c}_{F}=1-\frac{\Theta_{I}}{\mu^{2} \Theta_{F}}(\mu-1) .
$$

There is general qualitative agreement with data in the behaviour of the calculated binding energy of the ground states of $S=-1$ hypernucle1 with increasing atomic number, as can be seen from Tables 1,2 and Fig.4, but the binding energy is underestimated in most of cases.

The tendency of decrease of binding energies with increasing $B$-number, beginning with $B \sim 10$, is connected with the fact that the rational map approximation, leading to the one-shell structure of the classical configuration, is not good for such values of $B$.

\section{Binding energies of charmed or beautiful hypernuclei}

Same method can be applied for the prediction of the binding energies of charmed and beautiful hypernuclei [1]. Evident replacements should be made, $m_{K} \rightarrow m_{D}$ or $m_{B}$ and $F_{K} \rightarrow F_{D}$ or $F_{B}$.

For beautiful hypernuclei the binding energies are presented in Table 1 (isoscalar states), where the first 3 column correspond to $r_{b}=F_{B} / F_{\pi}=1.5$, and the last 3 - to $r_{b}=2$, and Table 2 (isodoublets, $r_{b}=2$ ).

For charmed hypernuclei the binding energies are presented in Table 3 [11. Without any new parameters, beautiful (or charmed) hypernuclei are predicted to be bound stronger than strange hypernuclei. Their binding energies only slightly depend on the poorly known values of the decay constants $F_{D}$ or $F_{B}[1]$. There is rough agreement of our results with some early estimates made within conventional (potential) approach first by C.Dover and S.Kahana [15] and later by several authors [16,17].

The model we used overestimates the flavour excitation energies, especially for strangeness, but is more reliable for differences of energies which contribute to the differences of binding energies we calculate here, and for charm or beauty quantum numbers. The binding energies of states with flavour quantum numbers $|F|=2$ or greater have been estimated roughly in [18].

\section{Theta-hypernuclei, strange, beautiful or charmed}

Situation with observation of exotic baryons remains to be somewhat contradictive (see, in particular, the talk by
Table 3. The binding energies of the charmed hypernuclei, isoscalars and isodoublets, with unit charm, $c=1 . \Delta \epsilon_{c}$ and $\epsilon^{\text {tot }}$ (both in Mev) are the same as in Tables 1,2, for the charm quantum number. The results are shown for two values of charm decay constant $F_{D}$, corresponding to $r_{c}=1.5$ and $r_{c}=2$ (the last 3 columns). The chemical symbol is ascribed to the nucleus according to its total electric charge.

\begin{tabular}{lllllll}
\hline$\Lambda A$ & $\omega_{c}^{r_{c}=1.5}$ & $\Delta \epsilon_{c}$ & $\epsilon_{c}^{t o t}$ & $\omega_{c}^{r_{c}=2}$ & $\Delta \epsilon_{c}$ & $\epsilon_{c}^{t o t}$ \\
\hline 1 & 1535 & - & - & 1673 & - & - \\
${ }_{\Lambda}^{3} \mathrm{He}$ & 1504 & 27 & 35 & 1647 & 24 & 32 \\
${ }_{\Lambda}^{5} \mathrm{Li}$ & 1505 & 25 & 52 & 1646 & 25 & 52 \\
${ }_{7} \mathrm{Be}$ & 1497 & 32 & 70 & 1641 & 30 & 68 \\
${ }_{9} \mathrm{~B}$ & 1518 & 11 & 68 & 1654 & 17 & 74 \\
${ }_{1}^{11} \mathrm{C}$ & 1525 & 4 & 79 & 1658 & 13 & 87 \\
${ }_{1}^{13} \mathrm{~N}$ & 1529 & 0 & 96 & 1660 & 10 & 106 \\
\hline
\end{tabular}

\begin{tabular}{llcllll}
\hline${ }_{\Lambda} A$ & $\omega_{c}^{r_{c}=1.5}$ & $\Delta \epsilon_{c}$ & $\epsilon_{c}^{t o t}$ & $\omega_{c}^{r_{c}=2}$ & $\Delta \epsilon_{c}$ & $\epsilon_{c}^{\text {tot }}$ \\
\hline${ }_{\Lambda}^{4} \mathrm{He}-{ }_{\Lambda}^{4} \mathrm{Li}$ & 1493 & 12 & 40 & 1639 & 16 & 44 \\
${ }_{\Lambda}^{6} \mathrm{Li}-{ }_{\Lambda}^{6} \mathrm{Be}$ & 1504 & 9 & 41 & 1646 & 14 & 46 \\
${ }_{\Lambda}^{8} \mathrm{Be}-{ }_{\Lambda}^{8} \mathrm{~B}$ & 1510 & 7 & 63 & 1648 & 15 & 71 \\
${ }_{1}^{10} \mathrm{~B}-{ }_{\Lambda}^{10} \mathrm{C}$ & 1520 & 0 & 65 & 1655 & 10 & 75 \\
${ }_{\Lambda}^{12} \mathrm{C}-{ }_{\Lambda}^{12} \mathrm{~N}$ & 1526 & -4 & 88 & 1659 & 7 & 99 \\
\hline
\end{tabular}

K.Hicks at present conference). Apparently, experimental methods of observation of relatively narrow resonances, with a width about $1 \mathrm{MeV}$ or smaller, need further development.

Same approach as in previous sections can be applied for the estimates of the binding energies of Theta- hypernuclei. For anti-flavour (positive strangeness, beauty or negative charm) the same formula as above holds, but with certain changes for the hyperfine splitting constants, $c_{F} \rightarrow c_{\bar{F}}$ and $\bar{c}_{F} \rightarrow \bar{c}_{\bar{F}}$ in the last term $\Delta M_{H F S} \cdot c_{\bar{F}}\left(\bar{c}_{\bar{F}}\right)$ is obtained from $c_{F}\left(\bar{c}_{F}\right)$ by means of substitution $\mu \rightarrow-\mu$ :

$$
c_{\bar{F}}=1-\frac{\Theta_{I}}{2 \mu \Theta_{F}}(\mu+1) ; \quad \bar{c}_{\bar{F}}=1+\frac{\Theta_{I}}{\mu^{2} \Theta_{F}}(\mu+1) .
$$

This change is crucially important for the link between rotator and bound state models of the $S U(3)$ quantization [20], but often it was not made in the literature. The mass of the $\Theta^{+}$hyperon within this approach equals to about $1570 \mathrm{MeV}\left(e=4.1, F_{K} / F_{\pi}=1.22\right)$.

As can be seen from Table 4, presenting some of the results obtained in 12 , the binding energies for Thetahypernuclei increase with increasing atomic number. If the $\Theta^{+}$pentaquark is not narrow and has the width of several tens of $\mathrm{MeV}$, as argued in 19, or even greater, the Thetahypernucleus can have much smaller width, and even be bound relative to the strong interactions 2. For rescaled (nuclear) variant of the model with smaller value of the

\footnotetext{
${ }^{2}$ It should be noted that there is a distinction between different quantization schemes in the next-to leading order of the $1 / N_{c}$ expansion for the flavour symmetry breaking terms in the spectrum of baryon states [20] - a problem not resolved yet consistently. The same holds for the widths of baryon resonances, see also [19.
} 
Table 4. The collective motion contributions to the binding energies of the Theta-hypernuclei with unit flavour, strangeness, charm or beauty, $S=+1, c=-1$ and $b=+1$. $\bar{\omega}_{s, c, b}$, in $M e v$, are the antiflavour excitation energies, $\epsilon^{t o t}$ is the total binding energy of the ground state of hypernucleus with $|F|=1$. For charm $r_{c}=1.5$, for beauty $r_{b}=2$.

\begin{tabular}{lllllll}
\hline$A$ & $\bar{\omega}_{s}$ & $\epsilon_{s}^{t o t}$ & $\bar{\omega}_{c}$ & $\epsilon_{c}^{t o t}$ & $\bar{\omega}_{b}$ & $\epsilon_{b}^{t o t}$ \\
\hline 1 & 591 & - & 1750 & - & 4940 & - \\
3 & 564 & 76 & 1710 & 46 & 4890 & 57 \\
5 & 558 & 108 & 1710 & 71 & 4880 & 82 \\
7 & 559 & 120 & 1710 & 85 & 4880 & 100 \\
9 & 550 & 152 & 1710 & 100 & 4900 & 100 \\
11 & 547 & 173 & 1710 & 115 & 4900 & 110 \\
13 & 546 & 196 & 1720 & 125 & 4910 & 120 \\
\hline
\end{tabular}

parameter $e$, which should be applied for larger atomic numbers, the binding energies of hypernuclei are greater. In view of these results being in qualitative agreement with more conventional approaches [21,22, 23], searches for such hypernuclear states are of interest.

\section{Conclusions}

Chiral soliton models, based on few principles and ingredients incorporated in the effective lagrangian, allow to describe qualitatively, in some cases quantitatively, various chracteristics of nuclei spectra - from ordinary $(S=0)$ nuclei to known light hypernuclei. The symmetry energy of nuclei with isospin up to 4 or $9 / 2$ is described for atomic numbers between 10 and 30 with only one fixed semifree parameter - Skyrme constant $e$.

The binding energies of the ground states of strange hypernuclei have been described in qualitative and in some cases quantitative agreement with data for atomic numbers up to $\sim 15$.

In view of this success, predictions of CSM are of interest, including the well bound heavy flavoured (charmed, beautiful) hypernuclei and so called Theta-hypernuclei, i.e. multibaryon states with positive strangeness or beauty, or negative charm.

Essential advantage of this approach is that the case of $B>1$, within CSA, does not differ in principle from the case of baryons, until the nonzero modes are included into consideration. There are some obvious drawbacks of this approach, as continuation of this advantage. In particular, one-, two-, etc. baryons excitations are not included - this is related to a very complicated problem of detailed study of nonzero modes of multiskyrmions. Specific and partly technical problem is also a smooth transition from the $B=1$ to rescaled "nuclear variant" of the model with smaller value of the parameter $e$.

Some scepticism concerning validity of the CSA - partly because of the unconfirmed narrow pentaquarks states has no firm grounds. Still, the chiral soliton approach is not the complete theory (of course!), but may carry some important features of the true theory.
The work is supported partly by the RFBR grant 0702-00960-a.

\section{References}

1. G. Adkins, C. Nappi and E. Witten, Nucl. Phys. B228, 552 (1983)

2. V. Kopeliovich, A. Shunderuk and G. Matushko, Phys. Atom. Nucl. 69, 120 (2006)

3. N. Manton and S. Wood, Phys.Rev. D74, 125017 (2006)

4. R.A. Battye and P.M. Sutcliffe, Rev. Math. Phys. 14, 29 (2002)

5. S.V. Zenkin et al, Sov.J.Nucl.Phys. 45, 106 (1987)

6. O. Manko and N. Manton, hep-th/0611179

7. R. Battye, N. Manton and P. Sutcliffe, Proc. Roy. Soc. Lond. A463, 261 (2007)

8. P. Irwin, Phys.Rev. D61, 114024 (2000)

9. S. Krusch, Annals Phys. 304, 103 (2003); Proc. Roy. Soc.Lond. A462, 2001 (2006); hep-th/0610176

10. I.R. Klebanov and K.M. Westerberg, Phys.Rev. D53, 2804 (1996); ibid. D50, 5834 (2004)

11. V.B. Kopeliovich, JETP 96, 782 (2003); ibid. 93, 435 (2001); Nucl. Phys. A721, 1007c (2003)

12. V.B. Kopeliovich and A.M. Shunderuk, JETP 100, 929 (2005)

13. H. Bando, T. Motoba and J.Zofka, Int.J.Mod.Phys. 21, 4021 (1990)

14. O. Hashimoto and H. Tamura, Prog.Part.Nucl.Phys. 57, $564(2006)$

15. C. Dover and S. Kahana, Phys.Rev.Lett. 39, 1506 (1977)

16. H. Bando and M. Bando, Phys.Lett. B109, 164 (1982); B. Gibson et al, Phys. Rev. C27, 2085 (1983),

17. N. Starkov and V. Tsarev, Nucl.Phys. A450, 507 (1990) S. Bunyatov et al, Sov. J. Nucl. Phys. 23, 253 (1992)

18. V.B. Kopeliovich and W.J. Zakrzewski, JETP Lett. 69, 721 (1999); Eur.Phys.J. C18, 369 (2000)

19. H. Walliser and H. Weigel, Eur.Phys.J. A26, 361 (2005); H.Weigel, hep-ph/0610123 (2006)

20. V.B. Kopeliovich and A.M. Shunderuk, Phys.Rev. D73, 094018 (2006)

21. G.A. Miller, Phys.Rev. C70, 022202 (2004)

22. D. Cabrera et al, Phys.Lett. B608, 231 (2005); E. Oset et al, Nucl.Phys. A755, 503 (2005)

23. X.H. Zhong et al, Phys.Rev. C71, 015206 (2005); ibid. C72, 065212 (2005) 